\title{
CLIMB - A 3U CubeSat to Van Allen belt
}

\author{
Emmeric Vitztum, Kaarel Repän, Alexander Nemetz, \\ Martin Eizinger, Ekin Ecem Baspinar, \\ \& Alexandros Sousanis \\ Department, Aerospace Engineering \\ University of Applied Sciences Wiener Neustadt \\ Wiener Neustadt, Austria
}

\author{
Carsten Scharlemann \\ Head of Department, Aerospace Engineering \\ University of Applied Sciences Wiener Neustadt \\ Wiener Neustadt, Austria \\ carsten.scharlemann@fhwn.ac.at
}

\begin{abstract}
CLIMB is a $3 U$ CubeSat whose goal is to reach the Van Allen belt with the help of an advanced propulsion system. Usually, the Van Allen radiation belt is avoided by spacecraft due to its high radiation levels. CLIMB aims exactly for this region to conduct various measurements. The spacecraft's subsystems need to be designed in a way to cope with this rough environment and to ensure that even in case of failure or loss of the spacecraft, it still shall deorbit within 25 years, as required by the Austrian Space Law. Some of the systems of CLIMB have already flown and been qualified during the previous Austrian mission PEGASUS.
\end{abstract}

Keywords - Clean Space; CLIMB; CubeSat; electric propulsion; FEEP; IFM; magnetic field; orbital debris mitigation; radiation; re-entry; Van Allen belt

\section{INTRODUCTION}

The magnetic field of Earth acts as a shield against incoming radiation from the Sun, as well as from interstellar space: many charged particles that reach Earth are trapped in the magnetic field outside of the atmosphere in the form of rings [1, p. 25], and do not reach the ground. While this shielding effect is beneficial for life on ground, it is detrimental for life in space [1, p. 25] due to the higher absorbed dose [1, p. 44] that accumulates through exposure to the locally increased flux of charged particles [2, p. 214]. The ISS - and with it the main residence for humans in outer space - has an average altitude of approximately 400 kilometres [3]. At this altitude, it is below both of Earth's high-radiation-density rings, normally called the Van Allen radiation belts $[1$, p. 25].

However, many other spacecraft operate in or at least pass through these regions [4]. As a consequence, details about this environment are relevant for spacecraft design in terms of life time and radiation hardness in general.

Efforts have been made to quantify the radiation levels and size of the Van Allen belts [5]. The shapes of the belts follow the field lines of Earth's nearly dipolar magnetic field (see Fig. 1), forming toroidal regions of high fluxes of protons, electrons, and ionised atoms (most notably helium, nitrogen, and oxygen [1, p. 25]. These fluxes decrease exponentially towards the boundaries of the belts. They peak at approximately $10^{8}$ electrons and $10^{3}$ protons per square centimetre and second in the inner and $10^{6}$ electrons per square centimetre and second in

1 These values consider only electrons with more than 500 kiloelectronvolts and protons with more than 100 megaelectronvolts of energy. the outer belt [2, pp. 214-215]. ${ }^{1}$ These peaks appear at around 2,000 and 31,000 kilometres respectively, but high chargedparticle densities associated with the Van Allen belts have been predicted at altitudes as low as 500 kilometres near the equator and even lower in the South Atlantic Anomaly (SAA) - a region where Earth's magnetic field is unusually weak [5] [1, p. 25]. The AE-8 model predicts an upper limit of the outer belt at approximately 50,000 kilometres altitude [1, p. 28], but exact values vary from source to source (e.g. [6]). This uncertainty may be due to the temporal variations of the belts.

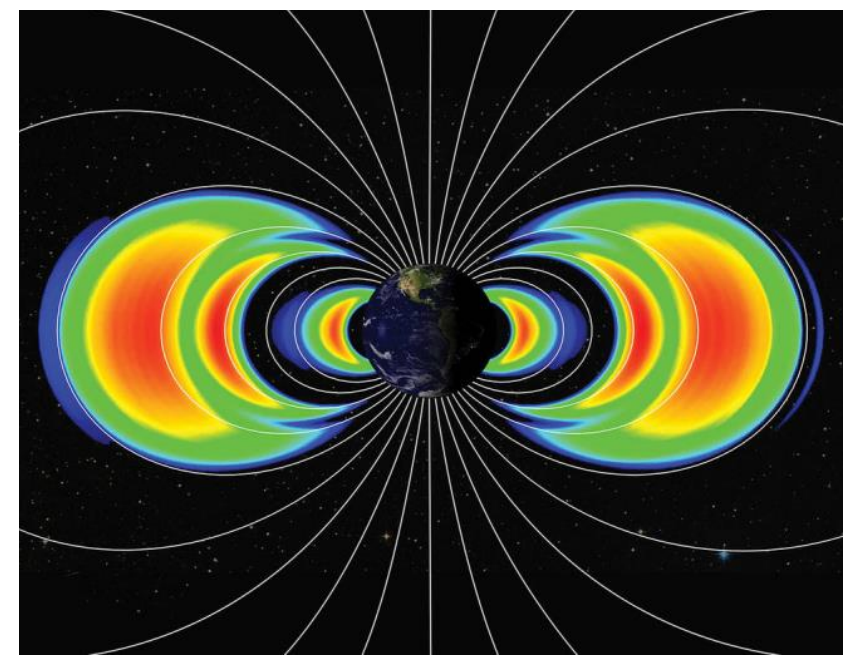

Fig. 1. Schematic visualisation of the Van Allen radiation belts. Their shapes result from the magnetic field lines (white), because magnetic interaction is the dominant force acting on the charged particles that constitute these belts. The image also shows the temporary third radiation belt. ${ }^{2}$ Credits: NASA's Goddard Space Flight Center/Johns Hopkins University, Applied Physics Laboratory

The academic satellite mission CLIMB aims to probe this environment using a design based on the CubeSat Design Specification. The mission combines commercial components with in-house designed solutions, while building on the experience of the mission PEGASUS (launched by the University of Applied Sciences Wiener Neustadt (FHWN) in

${ }^{2}$ The third radiation belt - a highly transient belt of electrons strongly dependent on solar activity - was first measured by the Van Allen Probes [10]. 
2017). The CubeSat Design Specification describes the volume of a spacecraft in terms of units, where one unit $U$ is a cube with a side length of 100 millimetres.

Despite existing measurements, the time-variability ${ }^{3}$ of the composition of the belts provides a permanent motivation for up-to-date data. The use of CubeSat components is one peculiarity of this mission compared to previous efforts (most notably NASA's Van Allen probes).

\section{MISSION OBJECTIVES}

As indicated above, the main scientific goal of the mission is to investigate the environment in the Van Allen belts in terms of electromagnetic radiation and charged particles. Another important objective is to prove that a CubeSat can survive such a challenging radiation environment without relying on extremely expensive radiation hardened components. Such solutions have already been successfully implemented in the former CubeSat PEGASUS resulting in an extremely low number of reboots or other radiation based anomalies [7].

In addition to that, a recently commercialised electric propulsion (EP) module - a crucial subcomponent - is demonstrated. The mission relies heavily on this component, which is used for reaching the target orbit as well as for active de-orbiting.

As a student project, education of participants as well as academic advance are noteworthy goals of CLIMB. In this context, it should be noted that the available resources only allow visiting the lowest regions of the inner Van Allen belt.

\section{MISSION DESCRIPTION}

The mission CLIMB uses a Field Emission Electric Propulsion (FEEP) system in order to reach the Van Allen belt and, upon arrival, conduct various measurements such as measurements of the magnetic field as well as of the radiation levels. Reaching the inner Van Allen belt by means of FEEP is considered a core part of the mission. As a consequence, the spacecraft operates in different orbits over the course of the mission. Fig. 2 shows the various orbits where the satellite operates, along with their associated mission phases.

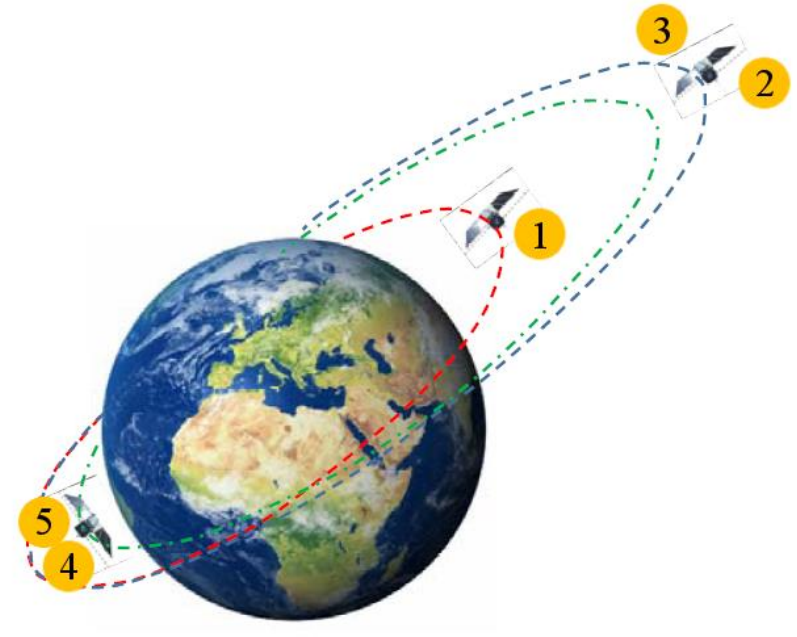

1 Launch and circular orbit

(2) Stepwise transfer into higher apogees

3 Mission in the Van Allen belt

(4) Stepwise transfer into lower perigees

5 Re-entry and burn-up

Fig. 2. Schematic depiction of the various phases in the CLIMB mission (orbits are not to scale)

\section{A. Launch and early operation (LEOP)}

Following the launch, the early operation phase is conducted. Most of the activities in this phase are pre-programmed including the deployment of the antennas and initiation of the beacon, automatic alignment of the satellite and basic functions of the thermal system.

\section{B. Commissioning phase}

During the commissioning phase a complete check of all systems shall assess their functionality. The outputs of all sensors shall be assessed and, if needed, calibrated. In case of anomalies, these shall be investigated to assess the probability and severity of their impact on the mission. Based on this assessment the decision is made whether to proceed with the mission or not.

\section{Orbit raising phase}

With the help of the propulsion system, the satellite increases its apogee while, in the early stage of this phase, leaving the perigee constant. In frequent steps, a health assessment of all relevant systems (propulsion, power, communication, etc.) is conducted. Only if all operational parameters are within the nominal range, the mission is allowed to proceed. When the

\footnotetext{
${ }^{3}$ Especially the heavy ion densities vary with solar and geomagnetic activity

$[1$, p. 25]
} 
apogee reaches a certain value, the satellite initiates a periodic decrease of the perigee while still increasing the apogee. Doing so ensures that the satellite would at any point naturally deorbit within 25 years, so as not to exceed the maximum allowable time in orbit. The final goal of the mission is an apogee with 1,000 kilometres altitude [8].

\section{Science phase}

The data that are relevant to the scientific objective of the mission (i.e. measurement of the magnetic field and radiation dose) are collected from the very beginning of the in-space operation. When CLIMB reaches the altitude of the Van Allen belt, the official science mission starts and the mission focuses on data collection rather than changing the orbit.

\section{E. De-orbiting phase}

In consideration of space situational awareness and the Austrian Space Law, specifically its requirements on orbital debris mitigation, many features have been implemented into the mission concept as well as in the technical design of CLIMB in order to ensure that the total time in orbit is well below 25 years - even in case of a loss of the spacecraft at any time during the mission. The Austrian Space Law requires that every space operator (coming from Austria) has to deorbit or remove ${ }^{4}$ their satellite at its EOL (end-of-life) from LEO (Low Earth Orbit). For non-manoeuvrable objects their post-mission lifetime shall not exceed 25 years.

Following the achievement of the scientific objectives, the satellite starts to actively deorbit. This is done in two ways:

- At the apogee, the propulsion system fires in retrograde direction to lower the spacecraft's perigee into the denser areas of the atmosphere.

- In the vicinity of the perigee the satellite aligns itself such that its maximum area (solar array) is perpendicular to the flight direction.

The mission ends with the satellite breaking up and burning up completely in the atmosphere.

\section{TECHNOLOGY}

Initially CLIMB was intended to be built upon the novel structure of the previous 2U CubeSat PEGASUS. Though, as both the dimensional and mass limitations were already exceeded early in the design phase of CLIMB, the decision was made to move on to a $3 \mathrm{U}$ CubeSat as seen in Fig. 3. Nevertheless, the structure is in essence identical to the one in PEGASUS. The main loads are carried by the separate rails in each corner of the CubeSat. The rails are connected by aluminium top and bottom panels and by the PCB (Printed Circuit Board) side panels. The internal PCBs are connected to the side panels only via electrical connectors, which therefore carry the mechanical loads. Some of the heavier subsystems (e.g. Attitude Determination and Control System, battery unit) also have additional aluminium attachment interfaces to the rails. The PCB side panels house the circuitry, which provides electrical interface between internal PCBs and other subsystems. The side panels also serve as a mechanical interface for the solar cells.

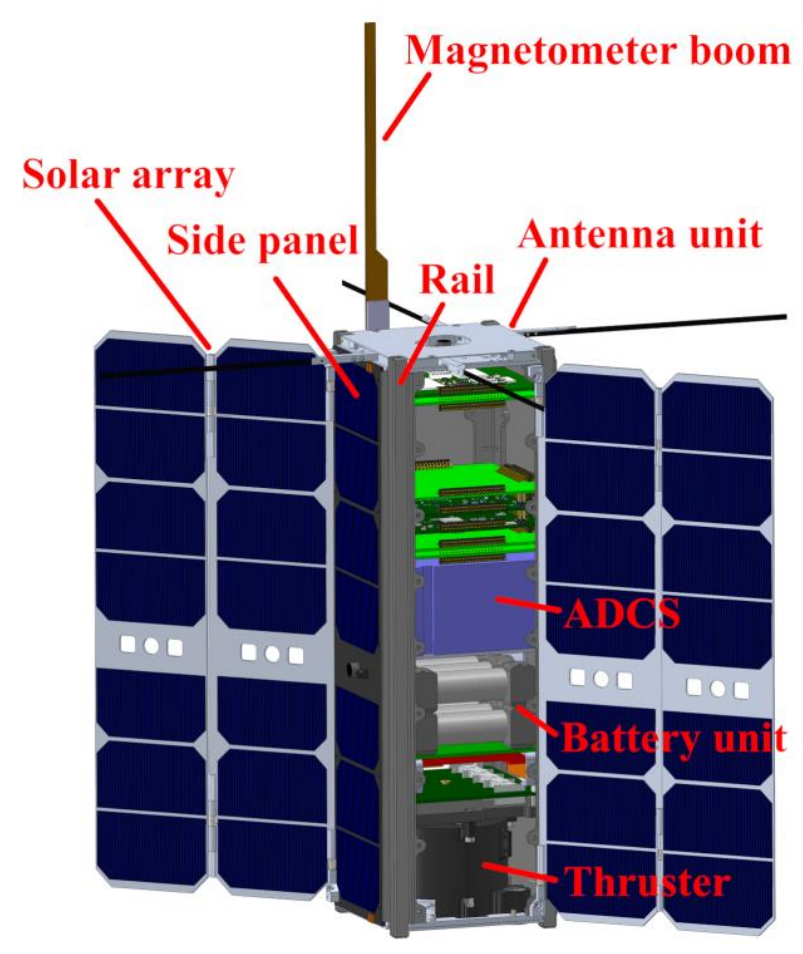

Fig. 3. General view of the CubeSat CLIMB without one side panel.

The main difference between the PEGASUS and CLIMB structures are the length of the rails and the side panels. Also, as the power requirement for CLIMB CubeSat is vastly increased - mainly because of the propulsion system - CLIMB incorporates deployable solar arrays. The thruster requires a maximum of 40 watts of electrical power whilst propelling and up to 5 watts during stand-by to keep the indium propellant in the liquid phase [9]. Also, other subsystems need at least a few watts of additional power. To provide this high amount of power, two double-folded deployable solar arrays are used in addition to the solar cells on the side panels: facing the Sun there are two sets of 7 solar cells on two side panels of CLIMB (nominally at 45 degree angle towards the Sun) and 2 solar arrays, each with 2 solar array elements (plates), each with 7 solar cells, totalling 28 deployable solar cells. The total power generated at peak is approximately 35 watts. For the hinges of the solar array, the usage of commercial fastenings is not possible because of the tight space requirements on the sides of the CubeSat - two packed solar array elements may occupy no more than 5 millimetres of space. The hinges of the solar arrays

\footnotetext{
${ }^{4}$ Putting the satellite into the graveyard orbit is allowed only if, for example the satellite is orbiting at GEO (Geostationary Earth Orbit).
} 
use the combination of laser welding of aluminium and soldering between aluminium hinge parts and PCB solar arrays.

The biggest subsystem is the Indium FEEP Multiemitter (IFM) Nano, provided by ENPULSION GmbH (shown in Fig. 4). The thruster has a wet mass of 900 grams and almost fills one CubeSat unit of volume. By default, the system comes with 230 grams of propellant, which is sufficient for the mission by a large margin. The propulsion system provides a nominal thrust of 350 micronewtons at a specific impulse of 4,000 seconds, with the ability to operate with less power at the cost of thrust or specific impulse. The second largest subsystem for CLIMB is expected to be the Attitude Determination and Control System (ADCS) as MAI-400 (from Adcole Maryland Aerospace, LLC) with a volume of $0.5 \mathrm{U}$ and a mass of 700 grams. It features three reaction wheels, a 3-axis magnetometer, two Infrared Earth Horizon Sensors (IREHS), three electromagnets and an ADCS computer for a stand-alone, plugand-play attitude control system.

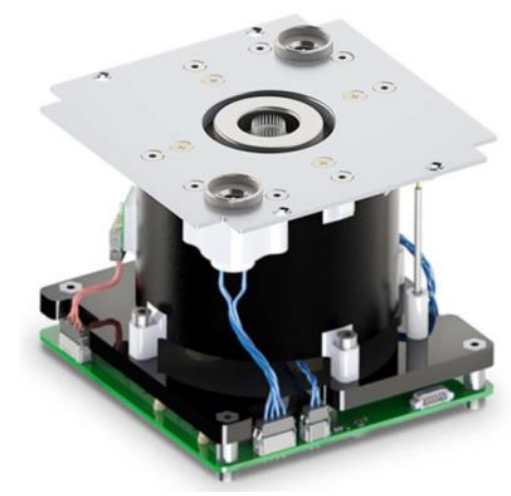

Fig. 4. Indium FEEP Multiemitter by ENPULSION GmbH [9]

Precise determination of the orbit is crucial for this type of mission. In order to support the ADCS, a laser ranging system is used. A laser on a ground station sends a high intensity laser pulse to the spacecraft. CLIMB is equipped with reflectors, to send back the laser pulse to the ground station. With the reflected signal, the position of the spacecraft and its orbit can be determined precisely. This helps to plan thrusting times and attitude corrections in a more efficient and reliable way compared to relying on the ADCS alone.

The following parts are inherited from the previous mission PEGASUS with minimal to no modifications or changes: inhouse developed OBC (On-Board Computer), PSU (Power Supply Unit) and STACIE-D (Space Telemetry And Command Interface - Delta), commercial off-the-shelf UHF (Ultra High Frequency) antennas, batteries and solar cells.

Two sets of battery units, each containing four 18650 type lithium ion rechargeable batteries, act as a buffer for electrical power (e.g. during eclipse times) and as a backup in case there are anomalies with the solar power generation. The already existing PSU needs to be adapted to the higher power requirements and the increased number of batteries. The outputs are 5 volts and 3.3 volts. Additional power converters located on the battery units are used to output 12 volts for the thruster. Two communication systems are used: the main one operating in the UHF- (STACIE-D) and the secondary in the S-band (STACIE-S).

All the individual thermal requirements for different subsystems must be satisfied. For example, if the temperature would drop below 0 degrees Celsius, the batteries would experience reduced capacity. As a countermeasure, the battery units are placed near the thruster to avoid the need for additional battery heaters. The waste heat from the thruster is directed towards the batteries. However, the batteries also exhibit a shortened lifetime with higher temperatures. If possible, the thermal design would keep the temperature of the batteries between 0 and 20 degrees Celsius.

As the power usage and thus power dissipation is relatively high for such a spacecraft size, a lot of effort is put into the thermal design. Various thermal elements are considered, including heaters, phase change materials, thermal straps, heat pipes, louvres, radiators, multi-layer insulation and different surface coatings. The highest power dissipation is expected to occur on the thruster and the battery unit (which includes converters for the thruster). Both of these subsystems are located at the bottom part of CLIMB. Another high power system, the S-band transceiver, is expected to dissipate about 8 watts of power. Though, as this subsystem is located at the top of the satellite, it might be less of a concern for the thermal configuration. At most, CLIMB is expected to dissipate about 26 watts of thermal power while thrusting.

The temperature ranges are highly dependent on the orbit. In the worst hot case with dawn-to-dusk orbit and calculated from the internal power dissipation, the preliminary estimate for the temperature is around 65 degrees Celsius with the highest temperatures occurring at the thruster.

During its journey and at its final destination in the Van Allen belt, CLIMB performs magnetic field measurements. For this purpose a deployable magnetometer boom is attached to the side of the spacecraft. The 25 centimetre long boom features analogue magnetic sensors at the tip and halfway to the tip. Close to the magnetic field sensors, temperature sensors are placed to measure the temperature and also to estimate thermal drift of the magnetic field sensors.

A series of in-house ground tests are performed. The available equipment includes a solar simulator, a Helmholtz coil, and a thermal vacuum chamber. Vibration tests are procured at FOTEC (Forschungs- und Technologietransfer GmbH), the scientific R\&D (Research \& Development) company owned by the University of Applied Sciences Wiener Neustadt.

Presently the CLIMB team focuses on the finalization of the satellite configuration. Firstly, for the solar arrays the preliminary design phase has been recently completed and mechanical (vibration) tests for the $2 \mathrm{U}$-configuration of the solar arrays have been carried out. Next step is to test the $3 \mathrm{U}$ configuration. Furthermore, more detailed numerical and 
experimental work is done with regard to the thermal design of CLIMB. These points are under current consideration and efforts are made to allow a launch date in early 2021.

\section{CONCLUSION}

The fulfilment of the scientific goal - i.e. obtaining measurement data of the space environment in the Van Allen belts - first requires the fulfilment of a technological goal: actively changing the orbit of the spacecraft. Orbital manoeuvres of the intended extent come with the well-known challenges of high specific impulse or a high propellant mass fraction. Achieving either of these on a CubeSat platform poses even greater difficulty. In that sense, CLIMB is pushing the boundaries of the state-of-the-art in nano-satellite technologies. Frequent design iterations have led to a $3 \mathrm{U}$ design that can accommodate even the larger and heavier subsystems like the MAI-400 ADCS and the IFM Nano propulsion system. Deployable solar arrays increase the irradiated area nearly fivefold in order to harvest enough power to supply the highly efficient EP system.

The CLIMB team has so far investigated various options for the configuration, achieved a detailed design, and specified all major subsystems. Several open points in the design still exist but will be tackled in the upcoming months.

\section{ACKNOWLEDGMENT}

This project is funded by the Lower Austrian Government and the University of Applied Sciences Wiener Neustadt. Very much appreciated is also the support of the CEO and scientific and technical staff of FOTEC. The CLIMB team also expresses its gratitude to ENPULSION $\mathrm{GmbH}$ for providing the propulsion system and sponsoring the trip to the symposium.

Furthermore, the team of the University of Applied Sciences Wiener Neustadt acknowledges the work of contributors, in particular the Space Tech Group Austria.

\section{REFERENCES}

[1] P. Fortescue, G. Swinerd and J. Stark, Spacecraft System Engineering, Chichester: Wiley, 2011.

[2] W. J. Larson and J. R. Wertz, Space Mission Analysis and Design, El Segundo, CA: Microcosm, 1999.

[3] esa.int, "international space station," 2019. [Online]. Available: https://www.esa.int/Our_Activities/Human_and_Robotic_Exploration/ International_Space_Station/Where_is_the_International_Space_Stati on. [Accessed 15 August 2019].

[4] ucsusa.org, "UCS Satellite Database," [Online]. Available: https://www.ucsusa.org/nuclear-weapons/space-weapons/satellitedatabase. [Accessed 15 August 2019].

[5] ccmc.gsfc.nasa.gov, “AE-8/AP-8 Radiation Belt Models," [Online]. Available: https://ccmc.gsfc.nasa.gov/modelweb/models/trap.php. [Accessed 15 August 2019].
[6] nasa.gov, "NASA's Van Allen Probes Spot an Impenetrable Barrier in Space," $2017 . \quad$ [Online]. Available: https://www.nasa.gov/content/goddard/van-allen-probes-spotimpenetrable-barrier-in-space.. [Accessed 15 August 2019].

[7] Scharlemann, C.; Seifert, B.; Schnitzer, R.; Kralofsky, R.; Obertscheider, C.; Taraba, M.; et al.; "PEGASUS - a review of in-orbit operation and obtained results," IAC-18,B4,3,3,x45633, IAC2018, Bremen, Germany, 2018.

[8] B. Weimer, C. Scharlemann, A. Reissner, D. Krejci and B. Seifert, "CLIMB: Exploration of the Van Allen Belt by CubeSats," IEPC-805, International Electric Propulsion Conference, Vienna, Austria, 2019.

[9] enpulsion.com, "IFM Nano Thruster," [Online]. Available: https://www.enpulsion.com/wp-content/uploads/ENP2018-001.FIFM-Nano-Thruster-Product-Overview.pdf. [Accessed 15 August 2019].

[10] nasa.gov, "The Van Allen Probes," 2018. [Online]. Available: https://www.nasa.gov/feature/goddard/2018/studying-the-van-allenbelts-60-years-after-america-s-first-spacecraft. [Accessed 15 August 2019]. 